\title{
CONJUGATION AND THE PRIME DECOMPOSITION OF KNOTS IN CLOSED, ORIENTED 3-MANIFOLDS
}

\author{
KATURA MIYAZAKI
}

\begin{abstract}
In this paper we consider the prime decomposition of knots in closed, oriented 3-manifolds. (For classical knots one can easily prove the uniqueness of prime decomposition by using a standard innermost disk argument.) We define a new relation, conjugation, between oriented knots in closed, oriented 3-manifolds and prove the following results. (1) The prime decomposition is, roughly speaking, uniquely determined up to conjugation, (2) there is a prime knot $\mathscr{R}$ in $S^{1} \times S^{2}$ such that $\mathscr{R} \# \mathscr{K}_{1}=\mathscr{R} \# \mathscr{K}_{2}$ if $\mathscr{K}_{1}$ is a conjugation of $\mathscr{K}_{2}$, and (3) if a knot $\mathscr{K}$ has a prime decomposition which does not contain $\mathscr{R}$, then it is the unique prime decomposition of $\mathscr{K}$.
\end{abstract}

\section{INTRODUCTION}

In [4] it was proved that the prime decomposition of a knot in $S^{3}$ is uniquely determined. However, to my knowledge there have been no papers studying the prime decomposition of 3-dimensional global knots in the sense of [5], i.e. knots in 3-manifolds. (Cf. [6].) In this paper we will study the prime decomposition of an oriented knot in a closed, oriented 3-manifold. First we obtain:

Existence Theorem. A nontrivial knot $\mathscr{K}$ is primely decomposable if and only if a meridian of $\mathscr{K}$ is essential in the exterior of $\mathscr{K}$ (Theorem 2.1).

This follows from a standard innermost disk argument and so-called Haken's finiteness theorem. However, for studying the uniqueness or nonuniqueness we need a new relation, called conjugation (defined in $\S 3$ ), between oriented knots in closed, oriented 3-manifolds. This relation is essential for prime decompositions of knots because the decomposition is, roughly speaking, uniquely determined up to conjugation (Theorem 5.4). In particular we have the following results.

Uniqueness Theorem. Let $\mathscr{R}$ be the prime knot in $S^{1} \times S^{2}$ depicted below. If a knot $\mathscr{K}$ has a prime decomposition which does not contain $\mathscr{R}$, then it is the unique prime decomposition for $\mathscr{K}$ (Corollary 5.6).

Received by the editors October 21, 1987 and, in revised form, February 1, 1988.

1980 Mathematics Subject Classification (1985 Revision). Primary 57M99; Secondary 57M25.

Key words and phrases. Prime knot, prime decomposition of knot, Haken's finiteness theorem, conjugation of knot, inducing-pair, annulus theorem. 

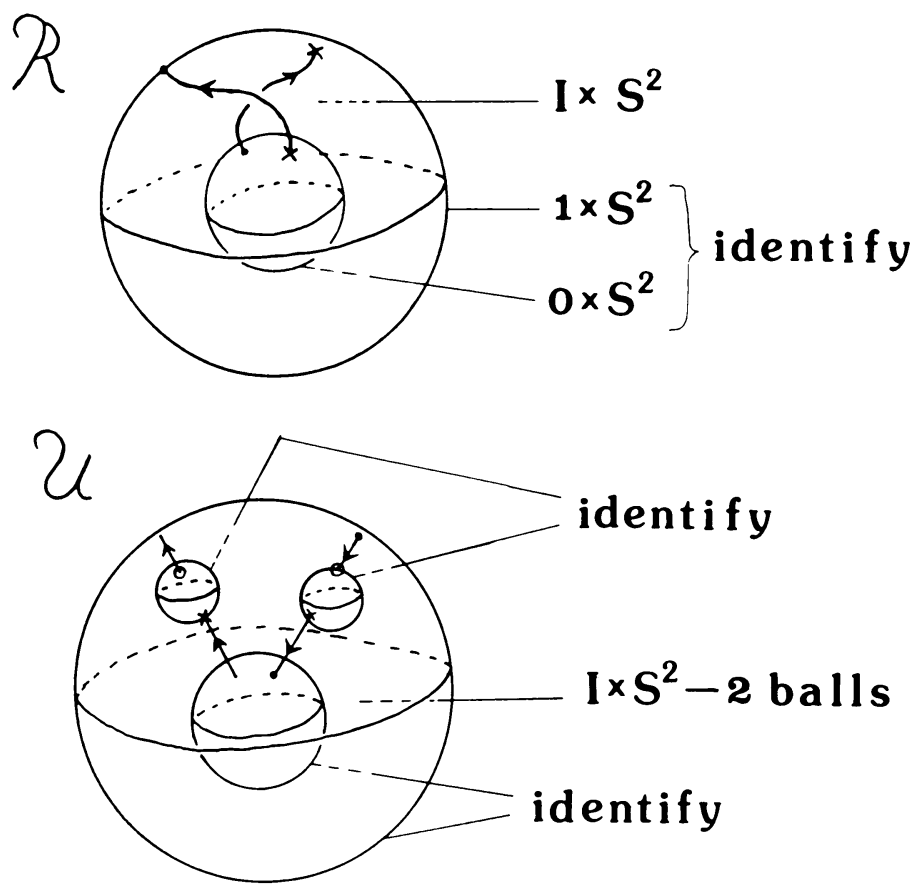

FIGURE 0

Nonuniqueness Theorem. If a knot $\mathscr{K}_{1}$ is a conjugation of a knot $\mathscr{K}_{2}$, then $\mathscr{R} \# \mathscr{K}_{1}=\mathscr{R} \# \mathscr{K}_{2}$ (Theorem 5.7). Let $\mathscr{U}$ be the prime knot in $2 S^{1} \times S^{2}$ shown in Figure 0 , then $\mathscr{R} \# \mathscr{R}$ is a conjugation of $\mathscr{U}$ (thus $\mathscr{R} \# \mathscr{R} \# \mathscr{R}=\mathscr{R} \# \mathscr{U}$ ) (Examples 2 and 3 in $\S 5$ ).

The paper is organized as follows. In $\S 1$ we establish terminology. In particular, we classify knots into two classes: order reversing knots and order preserving ones. In $\S 2$ we give a proof of the Existence Theorem (Theorem 2.1). The key idea of this paper is the notion of an inducing-pair introduced in $\S 3$. Using this, in $\S 3$ we define conjugation. $\S 4$ is devoted to the proof that every order preserving knot has a unique decomposition (Theorem 4.1). The lemma and the remark in $\S 4$ will be used in $\S 5$. In $\S 5$ first we study the order reversing knots. Then we clarify the rules of prime decomposition (Theorems 5.4-5.7).

I would like to thank Masahico Saito for suggesting this problem to me.

\section{Terminology}

We work throughout in the PL category, and assume that 3-manifolds are compact, connected and oriented.

A circle is a simple closed curve, and suitably oriented unless otherwise stated. For a circle $\alpha,-\alpha$ denotes the $\alpha$ with the orientation reversed. Two circles $\alpha_{1}, \alpha_{2}$ in a manifold $X$ are parallel if there exists an oriented annulus $A$ in $X$ with $\partial A=\alpha_{1} \cup-\alpha_{2}$. 
Let $M$ be a closed 3-manifold, and $K$ a circle in $M$. We call a pair $(M, K)$ a knot. We sometimes call the circle $K$ itself a knot. In this paper a capital letter in script (e.g. $\mathscr{K}, \mathscr{K}_{i}, \mathscr{R}$ etc.) stands for a knot, and when dealing with a knot $\mathscr{K}$, the circle of $\mathscr{K}$ is written as $K$ in italic type. The same notation applies to knots $\mathscr{K}_{i}, \mathscr{R}$, etc. Given a knot $\mathscr{K}=(M, K),-\mathscr{K}$ denotes the knot $(M,-K)$. Two knots $\left(M_{1}, K_{1}\right)$ and $\left(M_{2}, K_{2}\right)$ are said to have the same knot type if there is an orientation preserving homeomorphism $f:\left(M_{1}, K_{1}\right) \rightarrow\left(M_{2}, K_{2}\right)$. This is denoted by $\left(M_{1}, K_{1}\right) \cong\left(M_{2}, K_{2}\right)$.

Let $N(\cdots)$ denote a regular neighborhood. The exterior of a knot $\mathscr{K}=$ $(M, K)$ is $M-\operatorname{Int} N(K)$, which is denoted by $E(K)$ or $E(\mathscr{K})$. A meridian of a knot $(M, K)$ is a circle on $\partial N(K)$ which bounds on oriented disk $D$ in $N(K)$ with $D \cdot K=1$.

Let $N$ be a 3-manifold with $\partial N=S^{2}$, and let $a$ be an oriented interval properly embedded in $N$. We call a pair $(N, a)$ an $\operatorname{arc}$. For an arc $(N, a)$, $(A, a)^{-}$denotes the knot obtained from $(N, a)$ by capping the boundary pair $(\partial N, \partial a)$ with a trivial ball pair. Conversely, for a knot $\mathscr{K}=(N, a)^{\wedge} \mathscr{K}^{-}$ denotes the arc $(N, a)$. Two arcs $\left(N_{1}, a_{1}\right)$ and $\left(N_{2}, a_{2}\right)$ are said to have the same arc type if $\left(N_{1}, a_{1}\right)^{\wedge}$ and $\left(N_{2}, a_{2}\right)^{\wedge}$ have the same knot type.

For a 3-manifold $N$ with sphere boundaries, $\widehat{N}$ denotes the closed 3 manifold obtained from $N$ by capping $\partial N$ with 3-balls.

Given two knots $\mathscr{K}_{1}$ and $\mathscr{K}_{2}$, we define a connected sum

$$
\mathscr{K}_{1} \# \mathscr{K}_{2}=\left(N_{1}, a_{1}\right) \cup_{f}\left(N_{2}, a_{2}\right) \text {, }
$$

where $\mathscr{K}_{i}^{-}=\left(N_{i}, a_{i}\right)$ and $f:\left(\partial N_{2}, \partial a_{2}\right) \rightarrow\left(\partial N_{1}, \partial a_{1}\right)$ is an orientation reversing homeomorphism. For a knot $(M, K) \cong \mathscr{K}_{1} \# \cdots \# \mathscr{K}_{n+1}$ there exists a set of $n$ spheres in $M$ which gives this decomposition. When $n=1$, this follows from the definition of $\mathscr{K}_{1} \# \mathscr{K}_{2}$. Suppose $n-1$ spheres $S_{1}, \ldots, S_{n-1}$ gives a decomposition $\left(M^{\prime}, K^{\prime}\right)=\mathscr{K}_{1} \# \ldots \# \mathscr{K}_{n}$. We can isotop a trivial ball pair $(B, a)$ in $\left(M^{\prime}, K^{\prime}\right)$ so as to be disjoint from each $S_{i}$. Remove $(B, a)$ and attach $\mathscr{K}_{n+1}^{-}$we get the knot of the same knot type as $(M, K)$. The set of $n$ spheres $\left\{S_{1}, \ldots, S_{n-1}, \partial B\right\}$ gives the desired decomposition. Note that there is a choice of such a set.

Given a knot $\mathscr{K}=(M, K)$ and a closed 3-manifold $N$, define a connected sum $\mathscr{K} \# N=\left(M-\right.$ Int $B_{1} \cup_{f} N-$ Int $\left.B_{2}, K\right)$, where $B_{1} \subset M$ and $B_{2} \subset N$ are 3-balls missing $K$, and $f: \partial B_{2} \rightarrow \partial B_{1}$ is an orientation reversing homeomorphism.

A trivial knot in a 3-manifold is the connected sum of the unknot in $S^{3}$ and the 3-manifold.

A knot $\mathscr{K}$ is irreducible if $E(\mathscr{K})$ is an irreducible 3-manifold.

A knot $\mathscr{K}$ is prime if for each decomposition $\mathscr{K} \cong \mathscr{K}_{1} \# \mathscr{K}_{2}$ either $\mathscr{K}_{1}$ or $\mathscr{K}_{2}$ is the unknot in $S^{3}$, and $\mathscr{K}$ is not a trivial knot. Note that a prime knot is irreducible. A decomposition $\mathscr{K} \cong \mathscr{K}_{1} \# \ldots \# \mathscr{K}_{r} \# N$, where $\mathscr{K}_{1}, \ldots, \mathscr{K}_{r}$ are all prime and $N$ is a 3-manifold, is called a prime decomposition of $\mathscr{K}$. A 
knot which has a prime decomposition is said to be primely decomposable. All nontrivial knots in $S^{3}$ are primely decomposable.

Next we classify knots into two classes.

A knot $\mathscr{K}$ is said to be order reversing if they are disjoint, properly embedded annuli $A_{1}, A_{2}$ in $E(\mathscr{K})$ satisfying that:

(1) each component of $\partial A_{i}$ is a meridian;

(2) each component of $\partial E(\mathscr{K})-\partial A_{1}$ meets $\partial A_{2}$ in a circle. Otherwise, a knot $\mathscr{K}$ is said to be order preserving. The above $A_{1}$ and $A_{2}$ are called order reversing annuli in $E(\mathscr{K})$. We say an arc $(N, a)$ is order reversing (resp. order preserving) if a knot $(N, a)^{\wedge}$ is order reversing (resp. order preserving).

Let $X_{1}, X_{2}$ be 3-manifolds with boundaries, and $\alpha_{i}^{1}, \ldots, \alpha_{i}^{n}$ pairwise disjoint circles on $\partial X_{i}$. Choose a regular neighborhood $N_{i}^{j} \subset \partial X_{i}$ of $\alpha_{i}^{j}$ with $N_{i}^{j} \cap N_{i}^{k}=\varnothing \quad(j \neq k)$ and a homeomorphism $f: \bigcup_{j=1}^{n} N_{2}^{j} \rightarrow \bigcup_{j=1}^{n} N_{1}^{j}$ such that $f \mid:\left(N_{2}^{j}, \alpha_{2}^{j}\right) \rightarrow\left(N_{1}^{j}, \alpha_{1}^{j}\right)$ is orientation reversing for each $j$. The oriented homeomorphism class of $X_{1} \cup_{f} X_{2}$ is independent of the choice of $N_{i}^{j}$ and $f$. Let us denote $X_{1} \cup_{f} X_{2}$ by $X_{1} \cup_{\alpha_{1}^{j}=-\alpha_{2}^{j}} X_{2}$.

For a topological space $X$ we denote by $|X|$ the number of connected components of $X$.

We refer to [1 and 2] for a standard terminology in 3-manifold topology.

\section{EXISTENCE}

We will give a necessary and sufficient condition for a knot to be primely decomposable.

Theorem 2.1. A nontrivial knot $\mathscr{K}=(M, K)$ is primely decomposable if and only if a meridian $m \subset \partial E(K)$ is essential in $E(K)$.

Proof. Suppose that $m$ is null-homotopic. Let $\mathscr{K}=\mathscr{K}_{1} \# \cdots \# \mathscr{K}_{n} \# N$ be a prime decomposition. Now $\pi_{1}(E(K))=G$, say, is isomorphic to the amalgamated product

$$
G_{1}{ }_{\left[m_{1}\right]=\left[m_{2}\right]} G_{2} * \cdots * G_{n-1} *_{\left[m_{n-1}\right]=\left[m_{n}\right]} G_{n} * \pi_{1}(N),
$$

where $G_{i}=\pi_{1}\left(E\left(K_{i}\right)\right)$, and $\left[m_{i}\right]$ denotes some element in $G_{i}$ corresponding to a meridian of $\mathscr{K}_{i}$. If each [ $m_{i}$ ] generates an infinite cyclic subgroup in $G_{i}$, so does $[m]$ in $G$ by [3, Theorem 4.3], a contradiction. Thus we may assume that a meridian, $m_{1}$, of $\mathscr{K}_{1}$ represents a torsion or zero element in $G_{1}$. In other words for an annulus neighborhood $A\left(\subset \partial E\left(K_{1}\right)\right)$ of $m_{1} \operatorname{Ker}\left(\pi_{1}(A) \rightarrow G_{1}\right)$ is nontrivial. Then by the loop theorem [2, I.2] $m_{1}$ is null-homotopic in $E\left(K_{1}\right)$. By Dehn's lemma $m_{1}$ bounds a disk in $E\left(K_{1}\right)$, so that $\mathscr{K}_{1}$ admits a sphere intersecting $K_{1}$ transversely in a single point. Since $\mathscr{K}_{1}$ is an irreducible knot, $\mathscr{K}_{1} \cong\left(S^{1} \times S^{2}, S^{1} \times *\right)$. Then $\mathscr{K}_{1}$ can be expressed as the connected sum of $\mathscr{K}_{1}$ itself and any knot in $S^{3}$, contradicting the primeness of $\mathscr{K}_{1}$.

Suppose that a meridian of $\mathscr{K}$ is essential in $E(K)$. Then if $\left\{S_{1}, \ldots, S_{n}\right\}$ is a collection of spheres in $M$ which gives a decomposition $\mathscr{K} \cong \mathscr{K}_{1} \# \ldots \# \mathscr{K}_{n+1}$, $A_{i}=S_{i}-\operatorname{Int} N(K)$ is a properly embedded, incompressible annulus in $E(K)$. 
By III.24 in [2], there exists a nonnegative integer $N_{0}$ such that either $n<N_{0}$ or for some $i \neq j A_{i}$ is parallel to $A_{j}$ in $E(K)$. If the latter case occurs, then for some $i_{0} \mathscr{K}_{i_{0}}$ is a trivial knot. Thus $\mathscr{K}$ cannot be decomposed into more than $N_{0}$ nontrivial knots. Therefore, $\mathscr{K}$ can be decomposed into prime knots and a 3-manifold. This completes the proof of Theorem 2.1.

The next lemma reduces the prime decomposition problem to the case of irreducible knots. For a 3-manifold $M$, let $M^{-}$denote the 3-manifold obtained from $M$ by deleting an open 3-ball.

Lemma 2.2. Let $\mathscr{K}$ be primely decomposable. If $\mathscr{K}=\mathscr{K}_{1} \# \ldots \# \mathscr{K}_{n} \# N$ and $\mathscr{K}=\mathscr{K}_{1}^{\prime} \# \ldots \# \mathscr{K}_{m}^{\prime} \# N^{\prime}$ are prime decompositions, then $N=N^{\prime}, \#_{i=1}^{n} \mathscr{K}_{i}$ is an irreducible knot and $\#_{i=1}^{n} \mathscr{K}_{i} \cong \#_{i=1}^{m} \mathscr{K}_{i}^{\prime}$.

Proof. Set $X=E\left(\mathscr{K}_{1} \# \ldots \# \mathscr{K}_{n}\right)$. First we prove that $X$ is irreducible. Let $A_{1}, \ldots, A_{n-1}$ be properly embedded annuli in $X$ which are on the spheres giving the prime decomposition $\#_{i=1}^{n} \mathscr{K}_{i}=\mathscr{K}_{1} \# \cdots \# \mathscr{K}_{n}$. By the proof of Theorem 2.1 each $A_{i}$ is incompressible. If $X$ is reducible, then there is an incompressible sphere $S$ in $X$. Since each component of $X-\cup A_{i}$ is irreducible, $S$ may be isotoped so that $S \cap \bigcup_{i=1}^{n-1} A_{i}=\varnothing$. Hence, some $\mathscr{K}_{i}$ is not irreducible, contradicting the assumption. Similarly $X^{\prime}=E\left(\#_{i=1}^{m} \mathscr{K}^{\prime}\right)$ is irreducible. Let $N=\#_{i=1}^{s} N_{i}$ and $N^{\prime}=\#_{i=1}^{t} N_{i}^{\prime}$ be prime decompositions, and let $X \# N=\tilde{X}$, the exterior of $\mathscr{K}$. We then have two prime decompositions $\tilde{X}=X \# N_{1} \# \cdots \# N_{s}$ and $\tilde{X}=X^{\prime} \# N_{1}^{\prime} \# \cdots \# N_{t}^{\prime}$. Note that $X^{-}$and $X^{\prime-}$ are considered to be submanifolds of $\tilde{X}$ with $\partial \widetilde{X} \subset \partial X^{-}$and $\partial \widetilde{X} \subset \partial X^{\prime-}$. The following is proved in $[1,3.21]$ in the course of proving the unique decomposition theorem for 3-manifolds.

There is an orientation preserving homeomorphism $f: X^{-} \rightarrow X^{-}$with $f \mid \partial \widetilde{X}=$ id .

Thus $(M, K) \cong\left(M^{\prime}, K^{\prime}\right)$ and $N=N^{\prime}$.

Before going to the uniqueness problem we would like to notice the following.

Remark 2.3. The difficulty in showing the uniqueness of prime decomposition lies in the possible existence of nonseparating annuli properly embedded in $E(K)$ with their boundaries meridional. There are two cases:

(1) There are no such annuli. Then uniqueness can be proved in a standard manner.

(2) There exists such an annulus. Then two prime decompositions are related by conjugation. The uniqueness, however, holds if and only if every such annulus has oppositely oriented boundary components on $\partial N(K)$.

In the next section we define conjugation of knots.

\section{INDUCING-PAIRS}

Throughout this section we will use the following notation.

Let $X$ denote a 3-manifold with $\partial X=T_{1} \cup \cdots \cup T_{n}, n$ tori, and let $\alpha_{i} \subset T_{i}$ be an essential circle. 


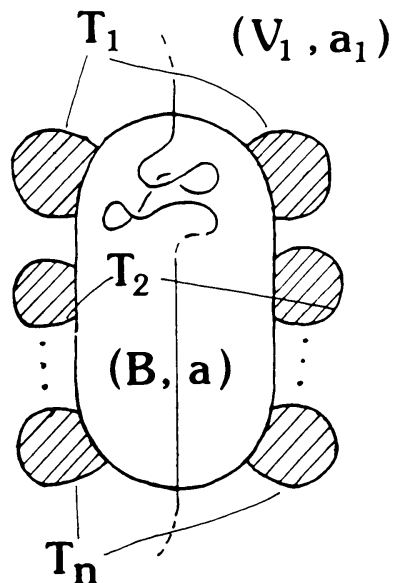

(a) The shaded area is $X$.

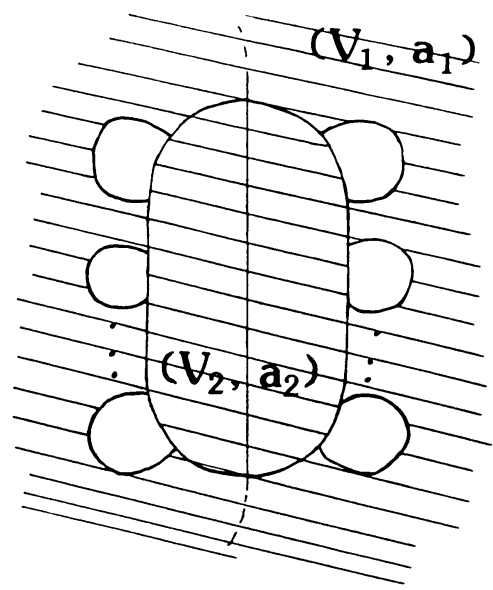

(b) The shaded area is $C_{n}$.

Figure 1. Cross sections

Let $(B, a)$ be an arc, $\beta_{1}, \ldots, \beta_{n} \subset \partial B-\partial a$ pairwise parallel, essential circles with $\mathrm{lk}_{\partial B}\left(\partial a, \beta_{i}\right)=+1$.

Let $(M, a)=X \cup_{\alpha_{i}=\beta_{i}}(B, a)$.

$D_{n}$ denotes a disk-with- $n$ holes with $\partial D_{n}=s_{0} \cup \cdots \cup s_{n}, n+1$ circles. $\left(C_{n}, J\right)$ denotes a pair $\left(S^{1} \times D^{2}, S^{2} \times 0\right) \cup_{f} D_{n} \times S^{1}$, where $f: s_{0} \times S^{1} \rightarrow S^{1} \times \partial D^{2}$ is a homeomorphism such that $f\left(p_{1} \times S^{1}\right)=p_{2} \times \partial D^{2}$ with $p_{1} \in s_{0}$ and $p_{2} \in S^{1}$.

The following lemma and the observations after that are essential for the present paper.

Lemma 3.1. If $(M, a)^{\wedge}$ is a prime knot, and $\left(X, \bigcup_{i=1}^{n} \alpha_{i}\right) \neq\left(S^{1} \times D^{2}, S^{1} \times *\right)$, $* \in \partial D^{2}$, then $B$ is a 3-ball and $(B, a)$ is a trivial ball pair.

Proof. Set $N=\partial X \cap B$ and $N^{\prime}=\mathrm{Cl}(\partial X-B)$. For $i=1,2$ let $\left(V_{i}, a_{i}\right)$ denote a trivial ball pair. Then

$$
\begin{aligned}
& (M, a)^{\widehat{ }}=\left(V_{1}, a_{1}\right) \cup_{(\partial M, \partial a)}(X \cup(B, a)) \quad(\text { cf. Figure 1(a) }) \\
& =\left(\left(V_{1}, a_{1}\right) \cup_{\cup N^{\prime}} X\right) \cup_{(\partial B, \partial a)}(B, a) \\
& =\left(\left(V_{1}, a_{1}\right) \cup \cup N^{\prime} X\right)^{\frown} \#(B, a)^{\uparrow} \\
& =\left(\left(V_{1}, a_{1}\right) \cup_{N^{\prime}} X \cup_{\cup N}\left(V_{2}, a_{2}\right)\right) \#(B, a)^{\wedge} \\
& =\left(X \cup_{T_{i}=s_{i} \times S^{1}}\left(C_{n}, J\right)\right) \#(B, a)^{\uparrow} \quad(\text { cf. Figure 1(b)) }
\end{aligned}
$$

Set

$$
\left(X^{\prime}, J\right)=X \cup_{T_{i}=s_{i} \times S^{\prime}}\left(C_{n}, J\right)
$$


Either $\left(X^{\prime}, J\right)$ or $(B, a)^{\wedge}$ must be the unknot in $S^{3}$, for $(M, a)^{\wedge}$ is a prime knot. When $n>1, X^{\prime}$ contains a nonseparating torus $T_{1}$, so that $(B, a)$ is trivial ball pair. If $n=1$ and $X^{\prime}=S^{3}$, then $J$ is knotted in $S^{3}$ by the assumption that $\left(X, \alpha_{i}\right) \neq\left(S^{1} \times D^{2}, S^{1} \times *\right)$, so that $(B, a)$ is a trivial ball pair. This completes the proof of Lemma 3.1.

In the rest of this section, $(B, a)$ denotes a trivial ball pair, but the assumption of the above lemma may not be satisfied.

Observaton 1. Now we have the following.

$$
(M, a)^{\wedge}=\left(X \cup_{\alpha_{i}=\beta_{i}}(B, a)\right)^{\wedge}
$$

and

$$
=X \cup\left(C_{n}, J\right) .
$$

The first equality follows from the definition of $(M, a)$. The union in $(* *)$ is given by $n$ orientation reversing homeomorphisms $f_{i}:\left(s_{i} \times S^{1},-l_{i}\right) \rightarrow\left(T_{i}, \alpha_{i}\right)$, $1 \leq i \leq n$, where $l_{i}=p_{i} \times S^{1}, p_{i} \in s_{i}$, is oriented so that $\mathrm{lk}_{C_{n}}\left(l_{i}, J\right)=1\left(C_{n}\right.$ is considered to be in $\left.S^{3}\right)$. Moreover, even if we interchange $\beta_{i}$ and $\beta_{j}, i \neq j$, in $(*)$, the resulting knot has the same knot type, for there is an orientation preserving autohomeomorphism $f$ of $\left(C_{n}, J\right)$ such that $f\left(l_{i}\right)=l_{j}, f\left(l_{j}\right)=l_{i}$, and $f \mid s_{k} \times S^{1}=$ id for $k \neq i, j$. Therefore, the $\operatorname{knot}(M, a)^{\wedge}$ and the arc $(M, a)$ depend only on $X$ and the circles $\alpha_{1}, \ldots, \alpha_{n} \subset \partial X$.

We say that a knot $\mathscr{K}=(M, a)^{\wedge}$ or an arc $(M, a)$ is induced from a pair $\left(X, \bigcup_{i=1}^{n} \alpha_{i}\right)$, and that $\left(X, \bigcup_{i=1}^{n} \alpha_{i}\right)$ is an inducing-pair of $\mathscr{K}$ with degree $n$. When $n=1$, it is called a trivial inducing-pair.

Lemma 3.2. Suppose that a prime knot $\mathscr{K}$ has a nontrivial inducing-pair with degree $n$. Then $E(K)$ contains $n$ incompressible tori dividing $E(K)$ into $D_{n} \times$ $S^{1}$ and another component.

Proof. Let $\left(X, \bigcup_{i=1}^{n} \alpha_{i}\right)$ be the inducing-pair in the assumption. From the equation (**) in Observation 1 we see $E(K)=X \cup D_{n} \times S^{1}$, where $\partial X$ is identified with $s_{i} \times S^{1}, 1 \leq i \leq n$. The torus $s_{i} \times S^{1}$ is incompressible in $D_{n} \times S^{1}$. Therefore if $\partial X$ were compressible in $E(K)$, there would be a properly embedded disk $D$ in $X$ with $\partial D$ essential in some $T_{i}$. Surgery on $T_{i}$ using $D$ yields a sphere in $X$. Since $n>1$, this sphere is nonseparating in $E(K)$, contradicting the primeness of $\mathscr{K}$. Hence, $\partial X$ is the desired tori.

A knot $\mathscr{K}$ is a conjugation of $\mathscr{K}^{\prime}$ through $\left(X, \bigcup_{i=1}^{n} \pm \alpha_{i}\right)$ if $\mathscr{K}$ is induced from $\left(X, \bigcup_{i=1}^{n} \alpha_{i}\right)$ and $\mathscr{K}^{\prime}$ is induced from $\left(X, \bigcup_{i=1}^{n} \alpha_{i}^{\prime}\right)$ where $\alpha_{i}= \pm \alpha_{i}^{\prime}$ up to order and isotopy in $\partial X$. Notice that $-\mathscr{K}$ is induced from $\left(X, \bigcup_{i=1}^{n}-\alpha_{i}\right)$. Similarly we can define conjugation for arcs.

It is immediate from the following observation that the ambient manifolds of $\mathscr{K}$ and $\mathscr{K}^{\prime}$ are homeomorphic to each other by an orientation preserving map if $\mathscr{K}$ is a conjugation of $\mathscr{K}^{\prime}$. 
Observation 2. There is another description of the arc $(M, a)$ induced from $\left(X, \bigcup_{i=1}^{n} \alpha_{i}\right)$. Divide $(B, a)$ into $2 n-1$ trivial ball pairs $\left(B_{i}, a_{i}\right), 1 \leq i \leq$ $2 n-1$, such that for $1 \leq i \leq n, B_{i}$ is attached to $X$ as a 2-handle along $\alpha_{i}$ (Figure 2). Then the boundary of $X \cup \bigcup_{i=1}^{n} B_{i}=X^{\prime}$, say, consists of $n$ spheres. For $n+1 \leq i \leq 2 n-1 \quad B_{i}$ is attached to $X^{\prime}$ as a 1-handle joining the distinct components of $\partial X^{\prime}$. It follows:

$$
(M, a) \cong X \cup_{\alpha_{1}, \ldots, \alpha_{n}} n\left(h^{2}, \text { cocore }\right) \cup(n-1)\left(h^{1}, \text { core }\right) .
$$

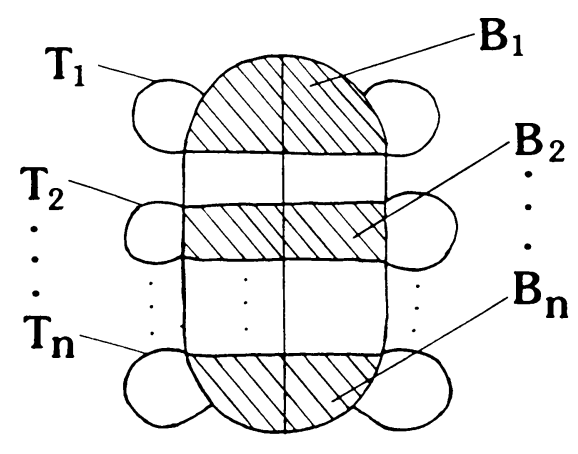

Figure 2. A cross section of $(M, a)$.

Hence, $\widehat{M} \cong \widehat{X}^{\prime} \#(n-1) S^{1} \times S^{2}$, and this is independent of the orientations of $\alpha_{i}$ 's. Now each cocore is oriented so that $\operatorname{lk}\left(\alpha_{i}\right.$, cocore $)=+1$. As long as 1handles are attached so that the obtained arc can be consistently oriented by the cocores, the resulting knot has the same knot type as $(M, a)^{-}$. If the attached 1 -handles do not satisfy the consistency of orientations, then the resulting knot is a conjugation of $(M, a)^{\wedge}$. Figure 3 illustrates the case where $\partial X$ consists of three tori.

\section{UNIQUENESS}

Unique prime decomposition holds for order preserving, primely decomposable knots.

Theorem 4.1. Let $\mathscr{K}$ be an order preserving, irreducible knot. If $\mathscr{K}=\mathscr{K}_{1} \# \ldots$ $\# \mathscr{K}_{n}$ and $\mathscr{K}=\mathscr{K}_{1}^{\prime} \# \cdots \# \mathscr{K}_{m}^{\prime}$ are prime decompositions, then $n=m, \mathscr{K}_{i} \cong \mathscr{K}_{i}^{\prime}$ up to order, and $\mathscr{K}_{i}$ and $\mathscr{K}_{j}^{\prime}$ are all order preserving knots.

Proof. It is easily checked that $\mathscr{K}_{i}$ and $\mathscr{K}_{j}^{\prime}$ are order preserving. We will prove the uniqueness by induction on $n$.

If $n=1$, then it is trivial. So, suppose $n>1$. Let $\mathscr{K}=(M, K)$, and let $\left\{S_{1}, \ldots, S_{n-1}\right\}$ be a collection of spheres giving the prime decomposition $\mathscr{K}=\#_{i=1}^{n} \mathscr{K}_{i}$. Let $\left(M_{i}, a_{i}\right), 1 \leq i \leq n$, denote the closure of each component of $(M, K)-\bigcup_{i=1}^{n-1} S_{i}$. We may assume that for each $i \mathscr{K}_{i} \cong\left(M_{i}, a_{i}\right)^{\wedge}$, the 

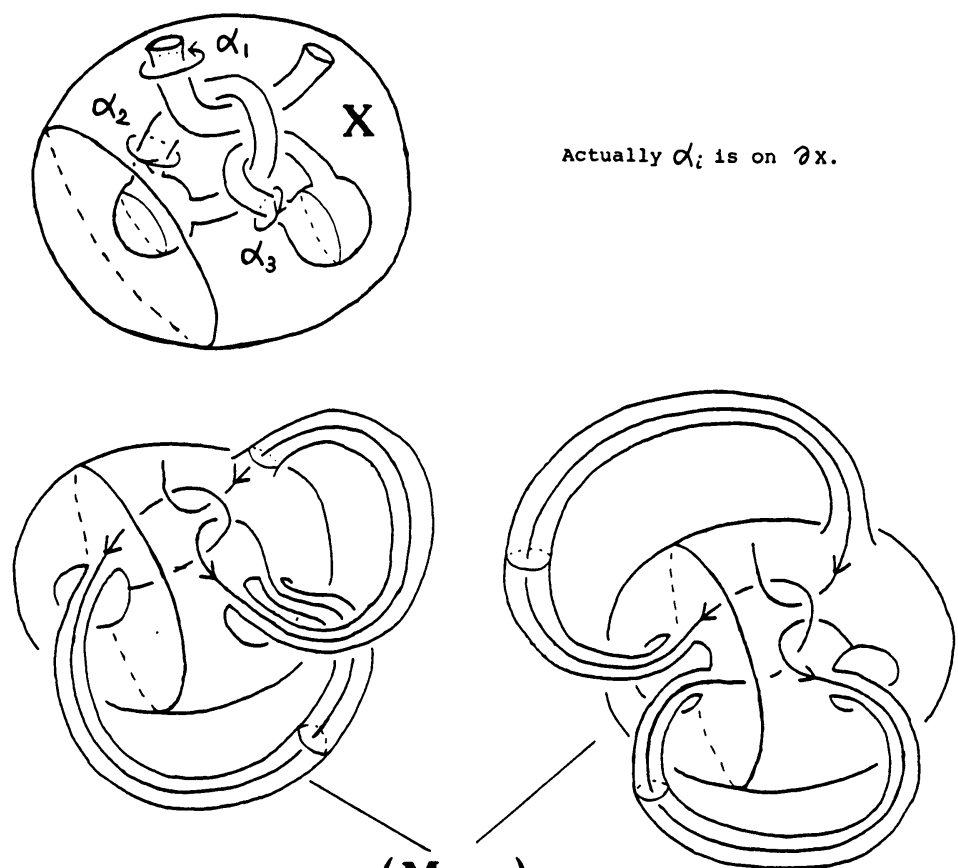

$(\mathbf{M}, \mathrm{a})$

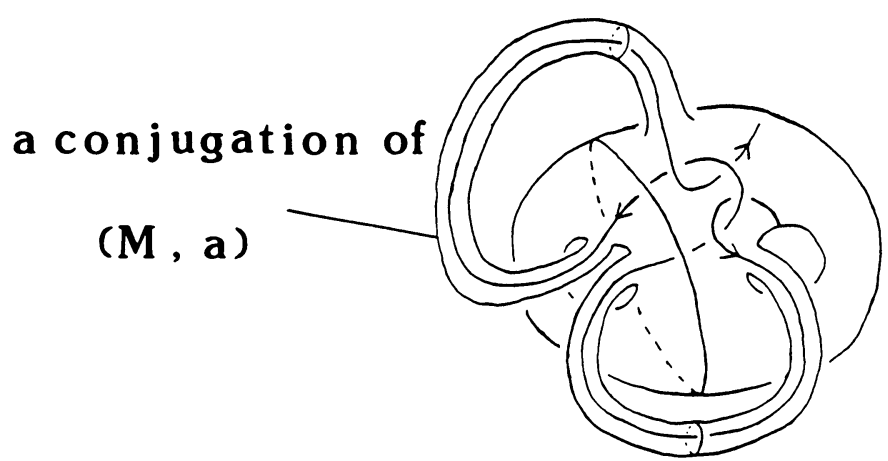

FIGURE 3

knot obtained from $\left(M_{i}, a_{i}\right)$ by capping $\left(\partial M_{i}, \partial a_{i}\right)$ with $\left|\partial M_{i}\right|$ trivial ball pairs. Suppose a collection of spheres $\left\{S_{j}^{\prime}\right\}_{j=1}^{m-1}$ gives the prime decomposition $\mathscr{K}=\#_{j=1}^{m} \mathscr{K}_{j}^{\prime}$. For this decomposition we define $\left(M_{j}^{\prime}, a_{j}^{\prime}\right), 1 \leq j \leq m$, similarly to $\left(M_{i}, a_{i}\right)$ above. Moreover, assume $\partial M_{n}=S_{n-1}$. If $M_{n} \cap \bigcup_{j=1}^{m-1} S_{j}^{\prime}=\varnothing$, then $\left(M_{n}, a_{n}\right)$ is contained in $\left(M_{j_{0}}^{\prime}, a_{j_{0}}^{\prime}\right)$ for some $j_{0}$, say $j_{0}=m$. From the primeness of $\mathscr{K}_{m}^{\prime}$ it is shown that $\mathscr{K}_{n} \cong \mathscr{K}_{m}^{\prime}$ and $\#_{i=1}^{n-1} \mathscr{K}_{i} \cong \#_{j=1}^{m-1} \mathscr{K}_{j}^{\prime}$. By induction the theorem is proved. Hence, we assume that $M_{n} \cap \bigcup_{j=1}^{m-1} S_{j}^{\prime}=\varnothing$.

Assertion 1. $\bigcup_{i=1}^{n-1} S_{i}$ and $\bigcup_{j=1}^{m-1} S_{j}^{\prime}$ can be isotoped separately with $\left|K \cap S_{i}\right|$ and $\left|K \cap S_{j}^{\prime}\right|$ fixed for each $i, j$ so that (1) each $S_{j}^{\prime}$ is transverse to $S_{n-1}$, 
(2) $M_{n} \cap \bigcup_{j=1}^{m-1} S_{j}^{\prime}$ is a nonempty set of annuli missing $K$, and (3) each boundary component of these annuli is essential in $S_{n-1}-K$.

Proof of Assertion 1. First isotop $\bigcup_{j=1}^{m-1} S_{j}^{\prime}$ with $\left|K \cap S_{j}^{\prime}\right|$ fixed for each $j$ so as to be disjoint from $a_{n}$. Next isotop $\bigcup S_{i}$ and $\bigcup S_{j}^{\prime}$ separately keeping $K \cap \bigcup S_{i}$ and $K \cap \bigcup S_{j}^{\prime}$ fixed so that $\bigcup S_{j}^{\prime}$ is transverse to $\bigcup S_{i}$ and $\left|\left(\bigcup S_{i}\right) \cap\left(\bigcup S_{j}^{\prime}\right)\right|$ is minimal. (If $M_{n} \cap \bigcup S_{j}^{\prime}=\varnothing$ after isotopy, the theorem is proved.)

We claim that $S_{j}^{\prime} \cap \bigcup_{i} S_{i}$ consists of essential circles in $S_{j}^{\prime}-K$ for each $j$. Part (2) of Assertion 1 follows from our claim. Suppose that for some $p$ $S_{p}^{\prime} \cap \bigcup S_{i}$ contains a circle null-homotopic in $S_{p}^{\prime}-K$. Then there is a disk $D^{\prime}$ such that Int $D^{\prime}$ is a component of $S_{p}^{\prime}-\bigcup S_{i}$ and $D^{\prime} \cap K=\varnothing$. The circle $\partial D^{\prime}$ bounds a disk $D$ on some $S_{q}$ with $\left|\left(D \cup D^{\prime}\right) \cap K\right| \leq 1$. Let $S$ denote the 2-sphere $D \cup D^{\prime}$, and let $s=|S \cap K|$.

If $s=0$, then $S$ bounds a 3-ball missing $K$ by the irreducibility of $\mathscr{K}$, so that the circle $\partial D^{\prime}$ is removed by isotoping $S_{q}$ without producing any new intersection. This contradicts the minimality assumption. If $s=1$, then $S$ is a 2-sphere meeting $K$ transversely in a single point, contradicting the existence of prime decomposition (Theorem 2.1).

Similarly we see that $S_{i} \cap \bigcup_{j} S_{j}^{\prime}$ consists of essential circles in $S_{i}-K$ for each $i$. So, (3) of Assertion 1 is proved.

Let $\left\{X_{1}, \ldots, X_{s}\right\}$ be the collection of the closures of components of $M_{n}-$ $\bigcup_{j=1}^{m-1} S_{j}^{\prime}$. The following is an immediate consequence of Assertion 1. (See Figure 4(a).)

Claim. (1) Every annulus component of $\partial M_{n} \cap X_{i}$ is essential in $\partial M_{n}-\partial a_{n}$, (2) if $X_{i} \cap a_{n}=\varnothing$, each component of $\partial X_{i}$ is a torus, and (3) there is just one $X_{i}$ such that $\partial X_{i}$ has a sphere component, which meets $K$ transversely in two points.

In order to apply Lemma 3.1 we need to modify $X_{i}, 1 \leq i \leq s$. Let $\left\{\partial_{1}, \ldots, \partial_{t}\right\}$ denote the set of the components of $\partial X_{i}$. Take an annulus $A_{j} \subset$ $\partial_{j}$ for $j=1, \ldots, t$ such that each of $A_{j} \cap\left(\partial X_{i} \cap \partial M_{n}\right)$ and $A_{j} \cap \mathrm{Cl}\left(\partial X_{i}-\partial M_{n}\right)$ is an annulus. (There is a choice of $A_{j}$.) Set $\widetilde{X}_{i}=\mathrm{Cl}\left(X_{i}-N\left(\left(\partial X_{i}-\bigcup_{j=1}^{t} A_{j}\right) \cup\right.\right.$ $\left.\left.a_{n}\right)\right)$. See Figure $4(\mathrm{~b})$. Let $\left\{\widetilde{\partial}_{1}, \ldots, \widetilde{\partial}_{t}\right\}$ denote the set of components of $\partial \widetilde{X}_{i}$, so that $\widetilde{\partial}_{j}$ is a torus for all $j$. Note that (1) $\left(\mathrm{Cl}\left(M_{n}-\widetilde{X}_{i}\right), a_{n}\right)$ is an arc for each $i,(2)$ the closure of $\widetilde{\partial}_{j}-\partial M_{n}$ is an essential annulus in both $\partial\left(M_{n}-\widetilde{X}_{i}\right)-\partial a_{n}$ and $\widetilde{\partial}_{j}$.

Assertion 2. There exists just one $i_{0} \in\{1, \ldots, s\}$ such that $\left(\widetilde{X}_{i_{0}}, \partial M_{n} \cap \widetilde{X}_{i_{0}}\right) \neq$ $\left(S^{1} \times D^{2}, S^{1} \times I\right)$, where $I$ is a subarc of $\partial D^{2}$.

Proof of Assertion 2. Suppose that both $\partial X_{i}$ and $\partial X_{j} \quad(i \neq j)$ satisfy the condition above. We apply Lemma 3.1 to $\widetilde{X}_{i}$ and $\left(\mathrm{Cl}\left(M_{n}-\widetilde{X}_{i}\right), a_{n}\right)$ to see 


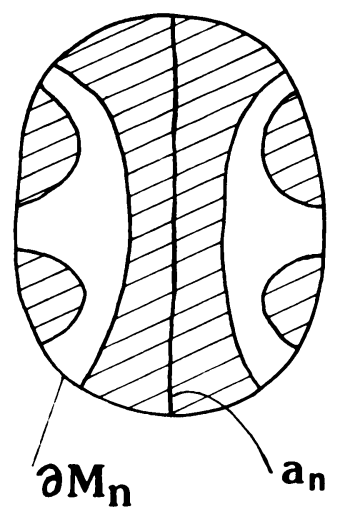

(a)

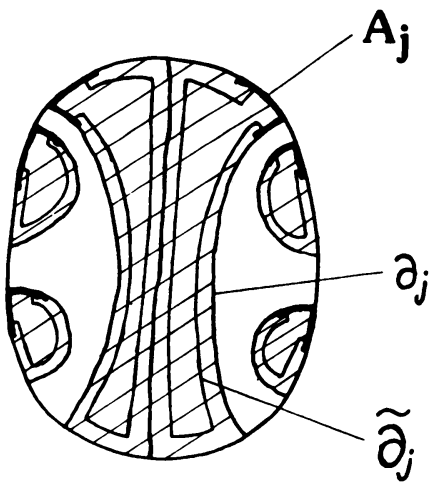

(b)

FIGURE 4. Cross sections of $\left(M_{n}, a_{n}\right)$.

The shaded area is some $X_{i}$.

the latter is a trivial ball pair. Thus $\left(\tilde{X}_{j}, \partial M_{n} \cap \widetilde{X}_{j}\right)=\left(S^{1} \times D^{2}, S^{1} \times I\right)$, contradicting the assumption.

If there exists $i_{0}$ such that $\partial X_{i_{0}}$ is disconnected, then $X_{i_{0}}$ is the desired component. Suppose that $\partial X_{i}$ is connected for all $i$. Let $\Omega$ denote $\left\{M_{n} \cap\right.$ ৩ $\left.S_{j}^{\prime}\right\}$, a set of annuli. We first claim that each $A \in \Omega$ separates $M_{n}$. Take an arbitrary element $A_{0}$ of $\Omega$. Let $B_{1}$ denote the annulus on $\partial M_{n}$ bounded by $\partial A_{0}$, and let $B_{2}=\mathrm{Cl}\left(\partial M_{n}-B_{1}\right)$. Define $\Omega_{j}=\left\{A \in \Omega \mid \partial A \subset B_{j}\right\}$, for $j=1,2$. Now $E\left(\mathscr{K}_{n}\right)=M_{n}-$ Int $N\left(a_{n}\right)$ does not contain order reversing annuli, thus $\Omega_{1} \cup \Omega_{2}=\Omega$ and $\Omega_{1} \cap \Omega_{2}=\left\{A_{0}\right\}$. Define $\Omega_{j}^{\prime}=\left\{i \mid \partial X_{i} \subset B_{j} \cup\right.$ the union of the elements of $\left.\Omega_{j}\right\}$, for $j=1,2$. We have $\Omega_{1}^{\prime} \cup \Omega_{2}^{\prime}=\{1, \ldots, s\}$, for $\partial X_{i}$ is connected for $i=1, \ldots, s$. Hence, we obtain

$$
M_{n}-A_{0}=\bigcup_{i=1}^{s} X_{i}-A_{0}=\left(\bigcup_{i \in \Omega_{1}^{\prime}} X_{i} \cup \bigcup_{i \in \Omega_{2}^{\prime}} X_{i}\right)-A_{0} .
$$

Since $\bigcup_{i \in \Omega_{1}^{\prime}} X_{i} \cap \bigcup_{i \in \Omega_{2}^{\prime}} X_{i}=\bigcup_{\Omega_{1}^{\prime}} \partial X_{i} \cap \bigcup_{\Omega_{2}^{\prime}} \partial X_{i}=A_{0}, M_{n}-A_{0}$ is disconnected as we claimed.

Suppose that each $X_{i}$ whose boundary is a torus satisfies $\left(\tilde{X}_{i}, \partial M_{n} \cap \widetilde{X}_{i}\right)=$ $\left(S^{1} \times D^{2}, S^{1} \times I\right)$. From the result just proved there exists an outermost annulus in $\Omega$, and each solid torus $X_{i}$ is attached to $\partial M_{n}$ with longitudinal annuli (i.e. regular neighborhood of longitudes). Remove all the solid tori $X_{i}$ 's from $M_{n}$ starting with the outermost ones, then the resulting arc has the same arc type as $\left(M_{n}, a_{n}\right)$, which is the union of $X_{i}^{\prime}$ such that $\left(X_{i}, X_{i} \cap a_{n}\right)$ is an arc. By (3) in Claim there is just one $i_{0}$ such that $\left(X_{i_{0}}, X_{i_{0}} \cap a_{n}\right) \cong\left(M_{n}, a_{n}\right)$. Since $\left(M_{n}, a_{n}\right)$ is not a trivial ball pair, $\left(\tilde{X}_{i_{0}}, \partial M_{n} \cap \widetilde{X}_{i_{0}}\right) \neq\left(S^{1} \times D^{2}, S^{2} \times I\right)$. This completes the proof of Assertion 2. 
Assertion 3. There exists $\left(M_{k}^{\prime}, a_{k}^{\prime}\right)^{\wedge}$ which is a conjugation of $\left(M_{n}, a_{n}\right)^{\wedge}$. For simplicity let us assume $k=m$, then $\#_{i=1}^{n-1} \mathscr{K}_{i} \cong \#_{j=1}^{m-1} \mathscr{K}_{j}^{\prime}$.

Proof of Assertion 3. Let $X_{i_{0}}$ be the component whose existence is guaranteed by Assertion 2. Let $\partial \widetilde{X}_{i_{0}}=T_{1} \cup \cdots \cup T_{r}, r$ tori. Applying Lemma 3.1 to $X_{i_{0}}$ and $\left(\mathrm{Cl}\left(M_{n}-\tilde{X}_{i_{0}}\right), a_{n}\right)=\left(B, a_{n}\right)$, say, we see that $\left(B, a_{n}\right)$ is a trivial ball pair. Take a core $\alpha_{i}$ of the annulus $T_{i} \cap B$ for $1 \leq i \leq r$. Orient $\bigcup \alpha_{i} \subset \partial B-\partial a_{n}$ by $a_{n}$ so that $\mathrm{k}_{\partial B}\left(\partial a_{n}, \alpha_{i}\right)=1$, then $\left(\widetilde{X}_{i_{0}}, \bigcup_{i=1}^{r} \alpha_{i}\right)$ is an inducing-pair of $\left(M_{n}, a_{n}\right)^{\text {. }}$.

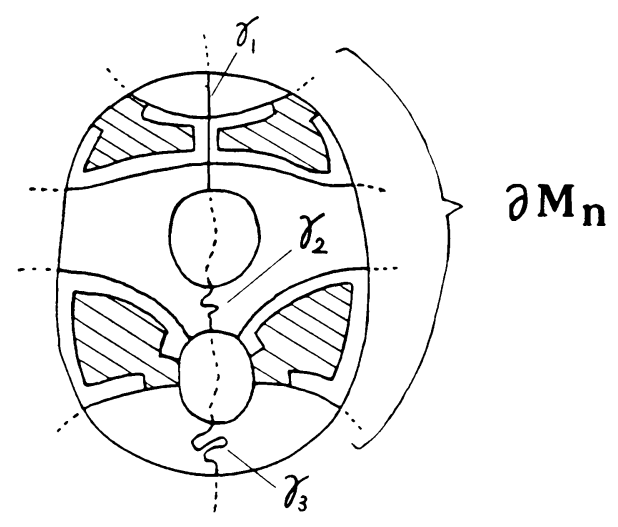

Figure 5. A cross section of $\left(M_{k}^{\prime}, a_{k}^{\prime}\right)$. The shaded area is $X_{i_{0}}$.

On the other hand, $\widetilde{X}_{i_{0}}$ is contained in just one $\left(M_{k}^{\prime}, a_{k}^{\prime}\right)$, where $a_{k}^{\prime}$ consists of arcs $\gamma_{1}, \ldots, \gamma_{u}, u=\left|\partial M_{k}^{\prime}\right|$, which are oriented by $K$. Note that for each $i T_{i}-\partial M_{k}^{\prime}$ is an open annulus whose core, $\beta_{i}$, is setwise iostopic to $\alpha_{i}$ on $T_{i}$. Hence, $\left(\mathrm{Cl}\left(M_{k}^{\prime}-\tilde{X}_{i_{0}}-\bigcup_{i=1}^{u-1} N\left(\gamma_{i}\right)\right), \gamma_{u}\right)$ is an arc, $\left(B^{\prime}, \gamma\right)$, say. The proof of Assertion 1 implies that each component of $\partial M_{k}^{\prime} \cap \partial M_{n}$ is essential in $\partial M_{k}^{\prime}-\partial a_{k}^{\prime}$, so that $\beta_{i}$ is essential in $\partial B^{\prime}-\partial \gamma$ (cf. Figure 5). Therefore, $\left(B^{\prime}, \gamma\right)$ is a trivial ball by Lemma 3.1. Orient $\bigcup_{i=1}^{r} \beta_{i} \subset \partial B^{\prime}-\partial \gamma$ by $\gamma$, then $\left(M_{k}^{\prime}, a_{k}^{\prime}\right)^{\wedge}=\left(\widetilde{X}_{i_{0}} \cup\left(B^{\prime}, \gamma\right)\right)^{\wedge}$ is induced from $\left(\widetilde{X}_{i_{0}}, \cup \beta_{i}\right)$. Since $\beta_{i}= \pm \alpha_{i}$ up to isotopy in $T_{i}$, the first part of Assertion 3 is proved.

Next we assume, without loss of generality, that $S_{m-1}^{\prime}$ is one of the boundary components of $\partial M_{k}^{\prime}$. Let $Y=M-\operatorname{Int} \widetilde{X}_{i_{0}}$ with $\partial Y=T_{1} \cup \cdots \cup T_{r}$. Paste $Y$ and $r$ solid tori $V_{1}, \ldots, V_{r}$ via $r$ homeomorphisms $f_{i}: T_{i} \rightarrow \partial V_{i}$, $1 \leq i \leq r$, such that $f_{i}\left(T_{i}-\partial M_{k}^{\prime}\right)$ is a longitudinal open annulus of $V_{i}$. Then we obtain $\left(M^{\prime \prime}, K^{\prime \prime}\right)=\mathscr{K}^{\prime \prime}$, where $M^{\prime \prime} \# \widehat{M}_{n} \cong M$ and $K=K^{\prime \prime}$. The spheres $\bigcup_{i=1}^{n-2} S_{i}$ and $\bigcup_{j=1}^{m-2} S_{j}^{\prime}$ remain in $\mathscr{K}^{\prime \prime}$ and give the prime decompositions $\mathscr{K}^{\prime \prime} \cong \mathscr{K}_{1} \# \cdots \# \mathscr{K}_{n-1}$ and $\mathscr{K}^{\prime \prime} \cong \mathscr{K}_{1}^{\prime} \# \cdots \# \mathscr{K}_{m-1}^{\prime}$, respectively. This establishes Assertion 3. 
Theorem 4.1 follows from Assertion 3 and the following assertion by induction on $n$.

Assertion 4. $\left(M_{k}^{\prime}, a_{k}^{\prime}\right)^{-} \cong\left(M_{n}, a_{n}\right)^{-}$.

We use the same notation as in the proof of Assertion 3. Suppose that $\left(M_{k}^{\prime}, a_{k}^{\prime}\right)^{\wedge} \not \equiv\left(M_{n}, a_{n}\right)^{\wedge}$. Then, there exists $l$ such that $\beta_{l}=-\alpha_{l}$ up to isotopy in $T_{l}$. Take an unoriented circle $C \subset T_{l} \cap \partial B \cap \partial B^{\prime}$ which is setwise isotopic to the circles $\alpha_{l}$ and $\beta_{l}$. Let $D$ be an oriented disk in $B$ such that $D$ meets the arc $a_{n}$ transversely in a single point with $D \cdot a_{n}=1$ and $D \cap \partial B=C$. Similarly define a disk $D^{\prime}$ and $B^{\prime}$. Since $\beta_{l}=-\alpha_{l}, D$ and $D^{\prime}$ give $C$ the opposite orientations. We may assume that $S=D \cup D^{\prime}$ is the oriented immersed sphere in $M$ with $S \cdot K=2$. Then, Assertion 4 will be proved by the following lemma and the assumption that $\mathscr{K}$ is order preserving.

Lemma 4.2. Suppose that $\mathscr{K}=(M, K)$ is irreducible and primely decomposable with $\mathscr{K}=\mathscr{K}_{1} \# \mathscr{K}_{2}$, where $\mathscr{K}_{2}$ is an order preserving knot. If $\mathscr{K}$ admits an oriented immersed sphere $S$ meeting $K$ transversely in two points with $S \cdot K$ $=2$, then $\mathscr{K}_{1}$ admits an embedded sphere meeting $K_{1}$ in the same way as $S$ meets $K$, so that $\mathscr{K}_{1}$ and $\mathscr{K}$ are order reversing.

To prove this lemma we will use the annulus theorem stated in [2, VIII.13]. Refer to [2] for the definitions of Haken manifold pairs, nondegenerate maps, and essential maps.

Proof of Lemma 4.2. Let $S^{\prime}$ be the sphere giving the decomposition $(M, K)=$ $\mathscr{K}_{1} \# \mathscr{K}_{2}$. Let $\left(M_{1}, a_{1}\right)$ and $\left(M_{2}, a_{2}\right)$ denote the closures of two components of $(M, K)-S^{\prime}$ with $\left(M_{i}, a_{i}\right)^{-}=\mathscr{K}_{i}$. The immersed sphere $S$ can be moved so as to meet $a_{1}$ transversely in two points by an ambient isotopy keeping $K$ setwise fixed.

Take some regular neighborhood $N(K)$ so that $S$-Int $N(K)$ is an immersed annulus, $A$, in $E(K)$ with $\partial A$ disjoint circles on $\partial E(K)$. Let $f:\left(S^{1} \times\right.$ $\left.I, S^{1} \times \partial I\right) \rightarrow(E(K), \partial E(K))$ be a map of pairs such that $f\left(S^{1} \times I\right)=A$ and $f \mid S^{1} \times \partial I$ is an embedding. If $\partial E(K)$ is compressible, then $\mathscr{K} \cong$ $\left(V_{1} \cup_{\partial V_{1}=\partial V_{2}} V_{2}, c\right)$, where $V_{1} \cong V_{2} \cong S^{1} \times D^{2}$, and $c$ is a core of $V_{2}$. Now $\mathscr{K}$ is primely decomposable, so $V_{1} \cup V_{2} \neq S^{1} \times S^{2}$. Since every 2-sphere in $V_{1} \cup V_{2}$ $\left(\neq S^{1} \times S^{2}\right)$ is separating, $\mathscr{K}$ does not admit $S$ satisfying the assumption of Lemma 4.2, a contradiction. We may assume that $\partial E(K)$ is incompressible, thus $(E(K), \partial E(K))$ is a Haken manifold pair. The map $f$ is nondegenerate because $f_{*}: \pi_{1}\left(S^{1} \times I\right) \rightarrow \pi_{1}(E(K))$ is injective and the assumption $S \cdot K \neq 0$ assures that $f$ is an essential map of pairs. By the annulus theorem there exists an embedding $h:\left(S^{1} \times I, S^{1} \times \partial I\right) \rightarrow(E(K), \partial E(K))$ with $h\left|S^{1} \times \partial I=f\right| S^{1} \times \partial I$. Let us denote the properly embedded annulus $h\left(S^{1} \times I\right)$ by also $A$. Note that $A$ gives a sphere whose intersection number with $K$ is two, or equivalently $A$ and one of the components of $\partial N(K)-\partial A$ form a Klein bottle. 
Now $A$ can be isotoped with $\partial A$ fixed so that $A \cap M_{2}$ are properly embedded annuli $A_{1}, \ldots, A_{n}$ whose boundary circles are essential in $S^{\prime}-K$, using the same argument as in the proof of Assertion 1. Let $B_{i}$ be the annulus on $S^{\prime}$ bounded by two circles $\partial A_{i}$. Since $\mathscr{K}_{2}$ is order preserving, $A_{i} \cup B_{i}$ is a torus for all $i$. If $B_{i} \cap B_{j} \neq \varnothing$, then either $B_{i} \subset B_{j}$ or $B_{j} \subset B_{i}$.

Modify $A$ as follows. Replace $A_{i}$ by $B_{i}$ for all $i$ and push into Int $M_{1}$ these annuli $B_{i}$ 's starting with the innermost ones (see Figure 6), then we get a properly embedded annulus in $M_{1}-\operatorname{Int} N(K)$. Since $A_{i} \cup B_{i}$ is a torus for all $i$, this annulus gives the sphere $S^{*}$ in $M_{1}$ such that $S^{*} \cdot K=2$. The two annuli $\partial N\left(S^{*}\right)-\operatorname{Int} N(K)$ are order reversing ones. This completes the proof of Lemma 4.2.

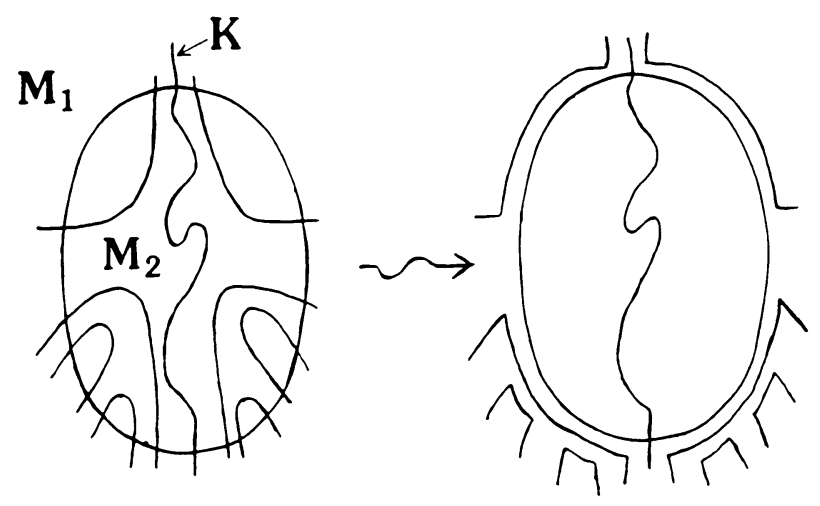

FIGURE 6

In proving Theorem 4.1 we also obtain the following, which will be used in the next section.

Remark 4.3. Suppose that $\mathscr{K} \cong \#_{i=1}^{n} \mathscr{K}_{i}$ and $\mathscr{K} \cong \#_{i=1}^{m} \mathscr{K}_{i}^{\prime}$ are prime decompositions, and some $\mathscr{K}_{i_{0}}$ is order preserving.

(1) Using the same argument as in the proof of Assertions 1-3, we get $\mathscr{K}_{j_{0}}^{\prime}$ a conjugation of $\mathscr{K}_{i_{0}}$ such that $\mathscr{K}_{1} \# \ldots \# \widehat{\mathscr{K}}_{i_{0}} \# \ldots \# \mathscr{K}_{n} \cong \mathscr{K}_{1}^{\prime} \# \ldots \# \widehat{\mathscr{K}}_{j_{0}}^{\prime} \# \ldots \# \mathscr{K}_{m}^{\prime}$ where the knots under $\wedge$ 's are deleted.

(2) If $\mathscr{K}_{i_{0}} \not \mathscr{K}_{j_{0}}^{\prime}$, then by the proof of Assertion $4 \mathscr{K}$ admits a sphere $S$ meeting $K$ transversely in two points with $S \cdot K=2$.

\section{NONUNIQUENESS}

First we consider typical examples of order reversing knots.

Example 1. A knot $\mathscr{R}$ is constructed from $\left(I \times S^{2}, I \times\left\{p_{1}, p_{2}\right\}\right)$, where $p_{1}, p_{2} \in$ $S^{2}$, by identifying $\{0\} \times S^{2}$ and $\{1\} \times S^{2}$ so as to yield a circle in $S^{1} \times S^{2}$. See Figure $7(a)$. It is easily checked that $\mathscr{R}$ is order reversing, prime, and $\mathscr{R} \cong-\mathscr{R} . E(R)$ is an annulus bundle over $S^{1}$ (in fact, a twisted $I$-bundle over a Klein bottle). 


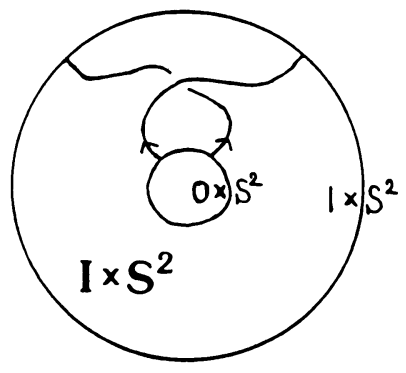

(a)
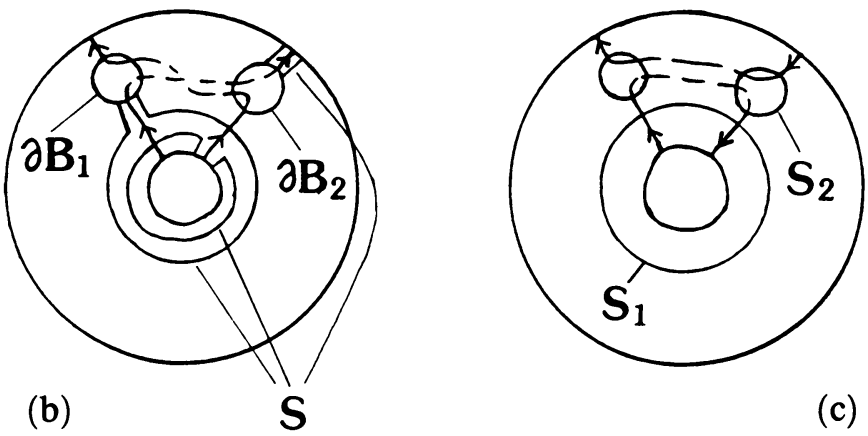

Figure 7

Example 2. Let $\left(X, \alpha_{1} \cup \alpha_{2}\right)=\left(S^{1} \times S^{1} \times I, S^{1} \times * \times\{0,1\}\right)$ where $\alpha_{1}$ and $\alpha_{2}$ are oriented to be parallel. The knot induced from $\left(X, \alpha_{1} \cup-\alpha_{2}\right)$ is constructed from $\left(S^{1} \times S^{2}, t_{1} \cup t_{2}\right)$, where $t_{i}=S^{1} \times p_{i}, i=1,2$, are oriented to be parallel. That is, first remove two disjoint 3-balls, $B_{1}$ and $B_{2}$, meeting $t_{1}, t_{2}$ respectively in an unknotted spanning arc. Then identify the resulting two boundary pairs $\left(\partial B_{i}, \partial B_{i} \cap t_{i}\right)$ via an orientation reversing homeomorphism to obtain the knot. This is because $\left(S^{1} \times S^{2}, t_{1} \cup t_{2}\right)-\operatorname{Int}\left(B_{1} \cup B_{2}\right)=X \cup_{\alpha_{1},-\alpha_{2}} 2\left(h^{2}\right.$, cocore $)$. This knot is $\mathscr{R} \# \mathscr{R}$. The sphere $S$ shown in Figure $7(\mathrm{~b})$ gives this decomposition. The fact that each summand is $\mathscr{R}$ is left to the reader as the exercise.

Example 3. With the notation of Example 2, the knot induced from $\left(X, \alpha_{1} \cup \alpha_{2}\right)$ is called $\mathscr{U}$. It is easily checked that the knot type of $\mathscr{U}$ is also induced from $\left( \pm X, \pm\left(\alpha_{1} \cup \alpha_{2}\right)\right)$, which are homeomorphic to each other via orientation preserving maps. $\mathscr{U}$ is order reversing, for a pair of $S_{1}$ and $S_{2}$ shown in Figure 7(c) give the order reversing annuli $S_{i}-\operatorname{Int} N(U)$ in $E(U)$. Recall Observation 1 of $\S 3$, then it follows $E(U)=X \cup D_{2} \times S^{1}=D_{1} \times S^{1} \cup D_{2} \times S^{1}$, where $D_{n}$ denotes a disk-with- $n$ holes as in $\S 3$. Inspecting the unions above, we see that $E(U)=\left(D_{1} \cup D_{2}\right) \times S^{1} \cong \stackrel{\circ}{T}^{2} \times S^{1}$, where $\stackrel{\circ}{T}^{2}$ is a torus with a hole. Under this homeomorphism from $\stackrel{\circ}{T}^{2} \times S^{1}$ to $E(U)$, a circle $* \times S^{1}$ in $\partial \stackrel{\circ}{T}^{2} \times S^{1}$ corresponds to a meridian of $\mathscr{U}$.

Lemma 5.1. $\mathscr{U}$ is prime. 
Proof. Identify $E(K)$ with $\stackrel{\circ}{T}^{2} \times S^{1}$ as above. If there is a sphere $S$ giving a nontrivial decomposition of $\mathscr{U}$, then $\stackrel{\circ}{T}^{2} \times S^{1}$ contains a separating, non- $\partial$-parallel, properly embedded annulus $A=S-\operatorname{Int} N(U)$ such that each component of $\partial A$ is setwise isotopic to $* \times S^{1}$ in $\partial \stackrel{\circ}{T}^{2} \times S^{1}$. Using a standard argument, we see that such an annulus does not exist in $\stackrel{\circ}{T}^{2} \times S^{1}$. Hence $\mathscr{U}$ is a prime knot.

Proposition 5.2. All the order reversing prime knots are $\mathscr{R}$ and $\mathscr{U}$.

Proof. Let $(M, a)$ be an arc with $(M, a)$ order reversing and prime. Let $A_{1}$ and $A_{2}$ be the order reversing annuli of $(M, a)$ as in Figure 8(1). For $i=1,2$ let $T_{i}$ denote the closed surface which is the union of $A_{i}$ and an annulus, $A_{i}^{\prime}$, on $\partial M-\partial a$ bounded by $\partial A_{i}$, so that $T_{i}$ is a Klein bottle or a torus.

Case 1. $T_{1}$ or $T_{2}$ is a Klein bottle. With no loss of generality we assume that $T_{1}$ is a Klein bottle. Let $(B, a)=\left(M-\operatorname{Int} N\left(T_{1}\right), a\right)$. Then $\partial B$ is a sphere and $B \cap N\left(T_{1}\right)$ is an annulus. See Figure 8(2). Applying Lemma 3.1 to $(B, a)$ and $N\left(T_{1}\right)$, we obtain $(B, a)$ is a trivial ball pair. Next let $S$ denote a sphere $\left(\partial M-A_{1}^{\prime}\right) \cup A_{1}$. See Figure $8(3)$. Then $M-\operatorname{Int} N(S) \cong B$ is a

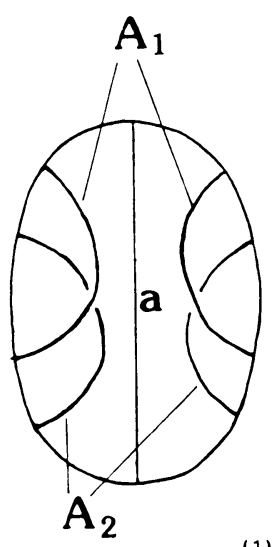

(1)

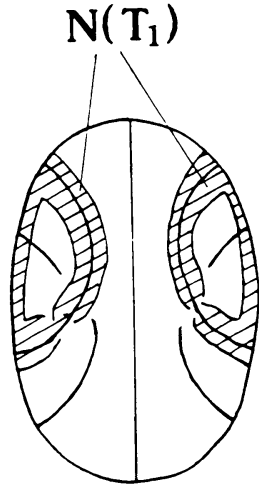

(2)

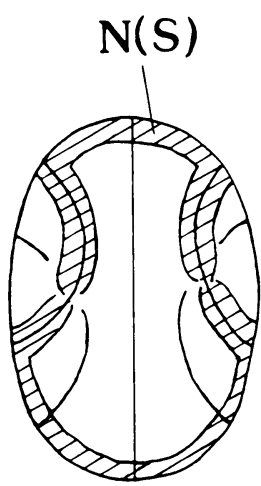

(3)

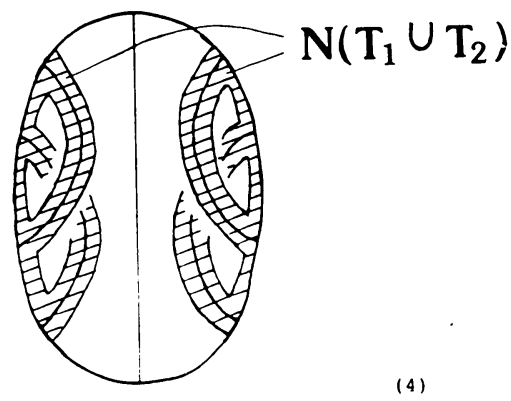

FIGURE 8 
3-ball, and $\partial M \cap(M-\operatorname{Int} N(S))$ is an essential annulus in $\partial M-\partial a$. Hence, $(M, a)^{-}-\operatorname{Int} N(S) \cong\left(I \times S^{2}, I \times\left\{p_{1}, p_{2}\right\}\right)$. More easily we have $(N(S), N(S) \cap$ $a) \cong\left(I \times S^{2}, I \times\left\{p_{1}, p_{2}\right\}\right)$. Example 1 shows that $(M, a)^{\wedge} \cong \mathscr{R}$.

Case 2. Each $T_{i}$ is a torus. Then each $N\left(T_{i}\right)$ is homeomorphic to $S^{1} \times S^{1} \times I$. Since $N\left(T_{1}\right) \cap N\left(T_{2}\right) \cong I \times I \times I$, it follows that $N\left(T_{1} \cup T_{2}\right) \cong \stackrel{\circ}{T}^{2} \times S^{1}$. (See Figure 8(4).) Applying Lemma 3.1 to $N\left(T_{1} \cup T_{2}\right)$ and $\left(M-\operatorname{Int} N\left(T_{1} \cup T_{2}\right), a\right)$, we see that the latter is a trivial ball pair, so that $N\left(T_{1} \cup T_{2}\right) \cong M-\operatorname{Int} N(a)$. Moreover, it follows that $\stackrel{\circ}{T}^{2} \times S^{1}$ is homeomorphic to $E((M, a) \wedge)$ with $* \times S^{1}\left(\subset \partial \stackrel{\circ}{T}^{2} \times S^{1}\right)$ corresponding to a meridian of $(M, a)^{\wedge}$. The observation in Example 3 shows that $(M, a)^{\wedge}=\mathscr{U}$.

Lemma 5.3. There exists no order preserving knot which is a conjugation of either $\mathscr{U}$ or $\mathscr{R}$.

Proof. If $\mathscr{R}$ had a nontrivial inducing-pair, $E(R)$ would contain an incompressible, nonseparating torus by Lemma 3.2. On the other hand, an incompressible torus in an annulus bundle over $S^{1}$ is $\partial$-parallel, so separating. Thus $\mathscr{R}$ itself is the only conjugation of $\mathscr{R}$.

We show that $\mathscr{U}$ has just one nontrivial inducing-pair given in Example 3, so that $\mathscr{R} \# \mathscr{R}$ and $\mathscr{U}$ are all the conjugation of $\mathscr{U}$. Let us identify $E(U)$ with $\stackrel{\circ}{T}^{2} \times S^{1}$ in the same way as in Example 3. It is easily checked that each incompressible torus in $\stackrel{\circ}{T}^{2} \times S^{1}$ is isotopic to $l \times S^{1}$ for some essential circle $l$ in $\stackrel{\circ}{T}^{2}$. If $E(U)$ contains incompressible tori, arbitrary two of them are parallel. Thus, by Lemma 3.2 a degree of inducing-pair of $\mathscr{U}$ is 1 or 2. Suppose that $\mathscr{U}$ has two inducing-pairs of degree 2. Let $c_{i} \times S^{1}(i=1,2)$ or $c_{j}^{\prime} \times S^{1}(j=1,2)$ be a pair of incompressible tori in $E(U)$ corresponding to each inducing-pair, where $c_{i}$ or $c_{j}^{\prime}$ is an unoriented circle in $\stackrel{\circ}{T}^{2}$. Since there is an orientation preserving homeomorphism $f: \stackrel{\circ}{T}^{2} \rightarrow \stackrel{\circ}{T}^{2}$ such that $f \mid \partial \stackrel{\circ}{T}^{2}=$ id and $f\left(\left\{c_{i}\right\}\right)=\left\{c_{j}^{\prime}\right\}$, these inducing-pairs are the same ones. This completes the proof of Lemma 5.3.

Here we are ready to state and prove our main theorems. A knot $n \mathscr{K}$ will denote $\mathscr{K} \# \ldots \# \mathscr{K}$ ( $n$ times).

Theorem 5.4. Suppose that $\mathscr{K}$ is primely decomposable. If

$$
\begin{aligned}
\mathscr{K} & \cong \mathscr{K}_{1} \# \ldots \# \mathscr{K}_{t} \# n \mathscr{R} \# m \mathscr{U} \# N \\
& \cong \mathscr{K}_{1}^{\prime} \# \ldots \# \mathscr{K}_{t^{\prime}}^{\prime} \# n^{\prime} \mathscr{R} \# m^{\prime} \mathscr{U} \# N^{\prime}
\end{aligned}
$$

are prime decompositions such that neither $\mathscr{K}_{i}$ nor $\mathscr{K}_{j}^{\prime} \cong \mathscr{R}$ or $\mathscr{U}$, then

(1) $t=t^{\prime} . \mathscr{K}_{i}^{\prime}$ is a conjugation of $\mathscr{K}_{i}$ up to order, $1 \leq i \leq t$. 
(2) $n+2 m=n^{\prime}+2 m^{\prime}$. Moreover, if $n=0$, then $n^{\prime}=0$, and $\mathscr{K}_{i} \cong \mathscr{K}_{i}^{\prime}$ up to order $1 \leq i \leq t$.

(3) $N \cong N^{\prime}$.

As immediate consequences we obtain:

Corollary 5.5. If $\mathscr{K}$ admits prime decompositions with distinct lengths, then every prime decomposition for $\mathscr{K}$ contains $3 \mathscr{R}$ or $\mathscr{U} \# \mathscr{R}$.

Corollary 5.6. If $\mathscr{K}$ has a prime decomposition which does not contain $\mathscr{R}$, then $\mathscr{K}$ does not admit any other prime decompositions.

Proof of Theorem 5.4. By Lemma $2.2 N \cong N^{\prime}$, thus we assume that $\mathscr{K}$ is irreducible. We will make some observations.

Case 1. $t>0$. By Remark 4.3 (1) and Lemma 5.3 for any $\mathscr{K}_{i}$ there exists $\mathscr{K}_{j}^{\prime}$ which is a conjugation of $\mathscr{K}_{i}$, and we have

$$
\mathscr{K}_{1} \# \ldots \# \widehat{\mathscr{K}}_{i}^{\#} \cdot . \# \mathscr{K}_{t} \# n \mathscr{R} \# m \mathscr{U} \cong \mathscr{K}_{1}^{\prime} \# \cdot . \# \widehat{\mathscr{K}}_{j}^{\prime} \# \cdot . \# \mathscr{K}_{t^{\prime}}^{\prime} \# n^{\prime} \mathscr{R} \# m^{\prime} \mathscr{U}
$$

where the knots under $\wedge$ 's are deleted. Suppose $n=0$. If $\mathscr{K}_{i} \not \mathscr{K}_{j}^{\prime}$, then by Remark 4.3 (2) and Lemma 4.2 there is a sphere $S$ in $2 m S^{1} \times S^{2}$ with $S \cdot m U=2$. This contradicts the fact that $m U$ is homologous to zero, so that $\mathscr{K}_{i} \cong \mathscr{K}_{j}$.

Case 2. $t=0$. Then,

$$
\mathscr{K} \cong n \mathscr{R} \# m \mathscr{U} \cong \mathscr{K}_{i}^{\prime} \# \ldots \# \mathscr{K}_{t^{\prime}}^{\prime} \# n^{\prime} \mathscr{R} \# m^{\prime} \mathscr{U} \text {. }
$$

By Remark 4.3 (1) $\mathscr{K}_{1}^{\prime}$ is a conjugation of either $\mathscr{R}$ or $\mathscr{U}$, contradicting Lemma 5.3. Therefore, $t^{\prime}=0$ and $n+2 m=n^{\prime}+2 m^{\prime}$. If $n=0$, then $n^{\prime}=0$, for the knot $m U$ is homologous to zero.

These observations and Remarks 4.3 (1) establish our theorem by induction on $t$.

In fact, the conclusion of Theorem 5.4 is best possible.

Theorem 5.7. If $\mathscr{K}_{1}$ is a conjugation of $\mathscr{K}_{2}$, then $\mathscr{R} \# \mathscr{K}_{1} \cong \mathscr{R} \# \mathscr{K}_{2}$. In particular, $\mathscr{R} \# \mathscr{R} \# \mathscr{R} \cong \mathscr{R} \# \mathscr{U}$.

Proof. Suppose that $\mathscr{K}_{1}$ is induced from $\left(X, \bigcup_{i=1}^{n} \alpha_{i}\right)$ and $\mathscr{K}_{2}$ is induced from $\left(X, \bigcup_{i=1}^{n} \alpha_{i}^{\prime}\right)$ with $\alpha_{i}$ and $\alpha_{i}^{\prime}$ on the same component of $\partial X$ for all $i$. Let $s=\#\left\{i \mid \alpha_{i}=-\alpha_{i}^{\prime}\right.$ up to isotopy in $\left.\partial X\right\}$. We will prove the theorem by induction on $s$.

If $s=0$, it is trivial.

If $s \geq 1$, then we may assume that $\alpha_{1}=-\alpha_{1}^{\prime}$, and let $\mathscr{K}_{3}$ be the knot induced from $\left(X,-\alpha_{1} \cup \bigcup_{i=2}^{n} \alpha_{i}\right)$. We claim that $\mathscr{R} \# \mathscr{K}_{1} \cong \mathscr{R} \# \mathscr{K}_{3}$, thus $\mathscr{R} \# \mathscr{K}_{1} \cong \mathscr{R} \# \mathscr{K}_{2}$ by induction.

Let $(B, a)$ be a trivial ball pair. Let $\beta_{1}, \ldots, \beta_{n}$ be pairwise parallel, essential circles on $\partial B-\partial a$ such that $\operatorname{lk}\left(a, \beta_{i}\right)=1$, and $\beta_{1}$ bounds a disk in $\partial B$ missing $\beta_{j}(j \neq 1)$, and such that there is an annulus $\omega$ in $\partial B$ with 
$(\mathrm{N}, \mathrm{b})$

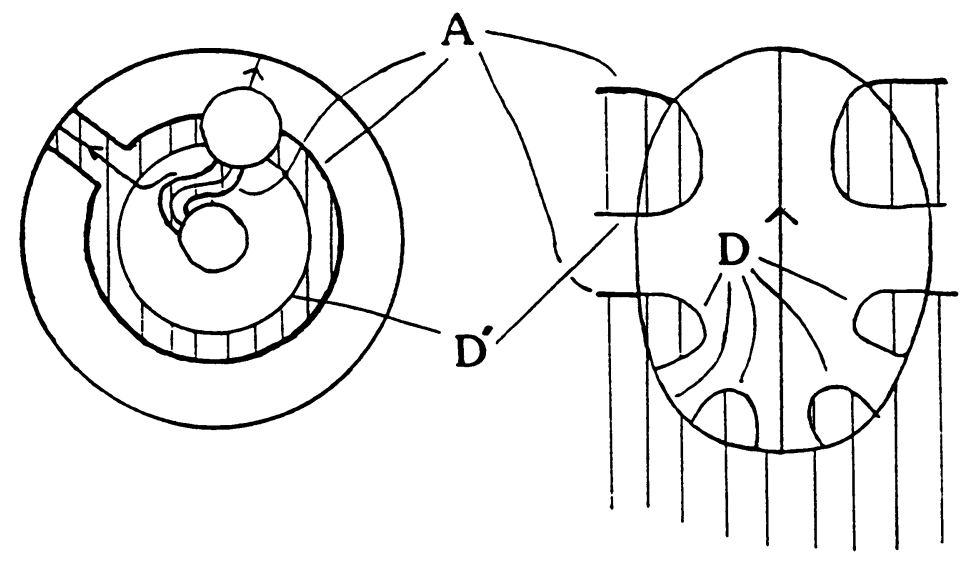

FIGURE 9

$\partial \omega=\beta_{1} \cup-\beta_{2}$ and $\beta_{i} \cap \omega=\varnothing(i \neq 1,2)$. Set $(M, a)=X \cup_{\alpha_{i}=\beta_{i}}(B, a)$, so $(M, a)^{\wedge} \cong \mathscr{K}_{1}$. Let $N\left(\beta_{i}\right)$ denote the annulus component of $X \cap B$ containing $\beta_{i}$. Let $D$ denote a disk in $\partial B$ such that $\partial D$ is one of the components of $\partial N\left(\beta_{2}\right)$ with $\beta_{2} \subset D$ and $\beta_{1} \cap D=\varnothing$.

Next we take a nonseparating disk $D^{\prime}$ properly embedded in the arc $\mathscr{R}^{-}=$ $(N, b)$ such that $D^{\prime}$ meets $b$ transversely in just one point, $\partial D^{\prime}$ is an essential circle in $\partial N-b$. Take a properly embedded annulus $A$ in $N-b$ such that $A$ and an annulus in $\partial N$ bounded by $\partial A$ form a Klein bottle, and $A \cap D^{\prime}=\varnothing$ (see Figure 9). Note that (a) $A \cup D^{\prime}$ separates $(N, b)$ into two trivial ball pairs $\left(B_{i}, a_{i}\right), i=1,2$, (b) $\partial B_{i} \cap \partial N$ consists of an annulus $u_{i}$ and a disk $v_{i}$, and (c) arbitrary essential circles $c_{1} \subset u_{i}$ and $c_{2} \subset v_{i}-b$ are parallel on $\partial B_{i}$ if and only if they are not parallel on $\partial N$.

We obtain $\mathscr{R} \# \mathscr{K}_{1}$ and the sphere $S=D \cup A \cup N\left(\beta_{1}\right) \cup D^{\prime}$ in $\widehat{M} \# S^{1} \times S^{2}$ by pasting $(M, a)$ and $(N, b)$ via an orientation reversing homeomorphism $f:(\partial N, \partial b) \rightarrow(\partial M, \partial a)$ with $f\left(\partial D^{\prime} \cup \partial A\right)=\partial N\left(\beta_{1}\right) \cup \partial D$. We may assume that $f\left(u_{1}\right) \subset \partial X$. Then $S$ separates $\mathscr{R} \# \mathscr{K}_{1}$ into two arcs. One is

$$
\left(B_{1}, a_{1}\right) \cup X \cong X \cup_{-\alpha_{1}, \alpha_{2}, \ldots, \alpha_{n}} n\left(h^{2}, \text { cocore }\right) \cup(n-1)\left(h^{1}, \text { core }\right) \cong \mathscr{K}_{3}^{-} ;
$$

another is $\left(B_{2}, a_{2}\right) \cup(B, a) \cong \mathscr{R}^{-}$, where $B_{2} \cap B=u_{2} \cup v_{2}$. Hence, $\mathscr{R} \# \mathscr{K}_{1} \cong$ $\mathscr{R} \# \mathscr{K}_{3}$. This completes the proof.

Remark. We can prove that an arbitrary 3-manifold contains a prime knot $\mathscr{K}$ with $\mathscr{K} \not-\mathscr{K}$. Combining this fact with Theorem 5.7, we obtain: every closed 3-manifold having $S^{1} \times S^{2}$ as a summand contains a knot which can be decomposed into prime knots in more than one way. 


\section{REFERENCES}

1. J. Hempel, 3-manifolds, Ann. of Math. Studies, no. 86, Princeton Univ. Press, Princeton, N.J., 1976.

2. W. Jaco, Lectures on three-manifold topology, CBMS Regional Conference Series in Math., no. 43, Amer. Math. Soc., Providence, R.I., 1980.

3. W. Magnuss, A. Karrass, and D. Solitar, Combinatorial group theory, Wiley, New York, 1966.

4. H. Schubert, Die eindeutige zerlegbarkeit eines knots in Primknoten, S. B.-Heidelberger Akad. Wiss. Math. Natur. Kl. 3 (1949), 57-104.

5. I. Tamura, Fundamental theorems in global knot theory, University of Tokyo, 1983, preprint.

6. K. Yano, The support of global graph links, J. Math. Soc. Japan 37 (1985), 683-702.

Department of Mathematics, The University of Texas at Austin, Austin, Texas 78712 